Journal of Engineering Sciences, Assiut University, Vol. 38, No. 1, pp. 209 -219, January 2010.

\title{
DETERMINATION OF CONTROLLERS GAINS LIMIT USING THE MIKHAILOV STABILITY CRITERION
}

\author{
Awad I. Saleh; Mohamed M. M. Hasan and Noha M. M. Darwish \\ Department of Electrical Engineering, Faculty of Engineering, Assiut \\ University, Assiut, Egypt
}

(Received October 25, 2009 Accepted November 15, 2009)

\begin{abstract}
Mikhailov's criterion states that a real Hurwitz polynomial $\delta(s)$ of degree $n$ satisfies the monotonic phase increase, that is to say the argument of $\delta(j w)$ goes through $n$ quadrants as $w$ runs from zero to infinity. In this paper, we utilize the generalized Mikhailov criterion presented in [1] to give a solution to the problem of finding the stabilizing feedback gains for a given linear-time invariant plant having a P controller, a PI controller and a PID controller.
\end{abstract}

\section{INTRODUCTION}

Consider a real polynomial $\delta(s)$ of degree $n$. We say that $\delta(s)$ is Hurwitz if all roots lie in the open left half plane $(\operatorname{Re}[\mathrm{s}]<0)$. We utilize the generalized Mikhailov criterion $[1,2,3]$ to give a solution to the problem of feedback stabilization of a given linear-time invariant plant using a P controller, a PI controller and a PID controller. Even though this problem can be solved using classical approaches such as the Nyquist stability criterion and the Routh-Huriwtz criterion, it is not clear how to extend these methods to the more complicated cases where PI or PID controllers are involved.

\section{A CHARACTERIZATION OF ALL STABILIZING P, PI AND PID CONTROLLERS}

Consider the feedback control system shown in Fig. (1),

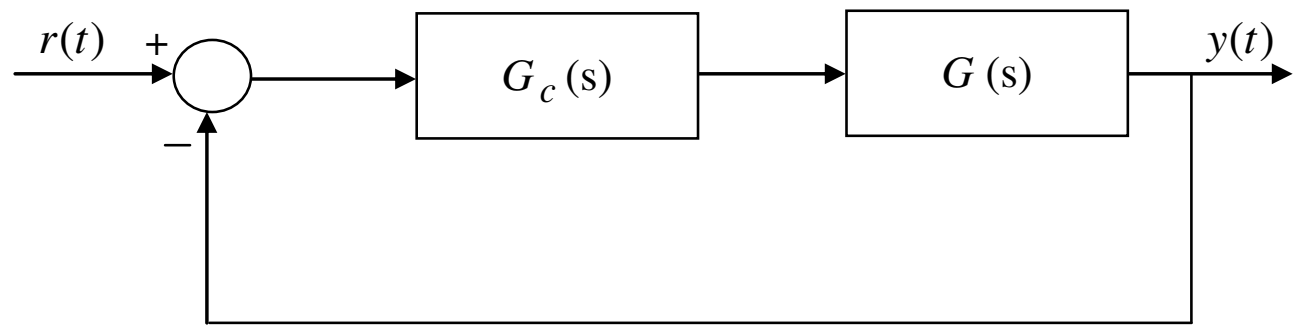

Fig. (1) Feedback control system

where $G(s)=\frac{N(s)}{D(s)}$ 
with $N(s), D(s)$ being coprime polynomials and degree $[N(s)] \leq$ degree $[D(s)]$.

The closed-loop characteristic polynomial $\delta(s)$ can be expressed as:

\section{- For a stabilizing P controller}

then

$$
G_{c}(s)=K_{p} \quad
$$

We can write

$$
\delta(s)=D(s)+K_{p} N(s)
$$

so that

$$
\begin{aligned}
& N(s)=N_{e}\left(s^{2}\right)+s N_{o}\left(s^{2}\right), \text { and } \\
& D(s)=D_{e}\left(s^{2}\right)+s D_{o}\left(s^{2}\right)
\end{aligned}
$$

$$
\delta(s)=\left[K_{p} N_{e}\left(s^{2}\right)+D_{e}\left(s^{2}\right)\right]+s\left[K_{p} N_{o}\left(s^{2}\right)+D_{o}\left(s^{2}\right)\right]
$$

It is clear that from this expression that both the even as well as the odd parts of $\delta(s)$ depend on $K_{p}$, which causes difficulties when trying to find the range of $K_{p}$ that makes the system stable. To overcome this problem, we will now construct a polynomial for which only the even part of this polynomial depends on $K_{p}[2,3]$.

Define

$$
N^{*}(s)=N(-s)=N_{e}\left(s^{2}\right)-s N_{o}\left(s^{2}\right)
$$

then

$$
\delta(s) N^{*}(s)=h_{1}\left(s^{2}\right)+K_{p} h_{2}\left(s^{2}\right)+s g_{1}\left(s^{2}\right)
$$

where

$$
\begin{gathered}
h_{1}\left(s^{2}\right)=D_{e}\left(s^{2}\right) N_{e}\left(s^{2}\right)-s^{2} D_{o}\left(s^{2}\right) N_{o}\left(s^{2}\right) \\
h_{2}\left(s^{2}\right)=N_{e}\left(s^{2}\right) N_{e}\left(s^{2}\right)-s^{2} N_{o}\left(s^{2}\right) N_{o}\left(s^{2}\right) \\
g_{1}\left(s^{2}\right)=N_{e}\left(s^{2}\right) D_{o}\left(s^{2}\right)-D_{e}\left(s^{2}\right) N_{o}\left(s^{2}\right)
\end{gathered}
$$

Substituting $s=j w$, we obtain

where

$$
\delta(j w) N^{*}(j w)=(R(w)+j I(w))\left(N_{R}^{*}(w)+j N_{I}^{*}(w)\right)
$$

$R(w)$ and $N_{R}^{*}(w)$ are real parts of $\delta(j w)$ and $N^{*}(j w)$ respectively.

$I(w)$ and $N_{I}^{*}(w)$ are imaginary parts of $\delta(j w)$ and $N^{*}(j w)$ respectively,

$$
\begin{aligned}
& N_{R}^{*}(w)=N_{e}\left(-w^{2}\right), \text { and } \\
& N_{I}^{*}(w)=-w N_{o}\left(-w^{2}\right)
\end{aligned}
$$

Then

$$
\begin{aligned}
\delta(j w) N^{*}(j w) & =\left(R(w) N_{R}^{*}(w)-I(w) N_{I}^{*}(w)\right)+j\left(R(w) N_{I}^{*}(w)+I(w) N_{R}^{*}(w)\right) \\
& =p(w)+j q(w)
\end{aligned}
$$


This last equation is a general form for any controller type.

If $\delta(s) N^{*}(s)$ has roots on the imaginary axis [1], then

$$
\delta(j w) N^{*}(j w)=0
$$

and as a result $p(w)=0$ and $q(w)=0$

So,

$$
\begin{aligned}
& R(w) N_{R}^{*}(w)-I(w) N_{I}^{*}(w)=0, \\
& \text { and } \quad R(w) N_{I}^{*}(w)+I(w) N_{R}^{*}(w)=0
\end{aligned}
$$

Equations (3), (4) will equal zero either if

i) $\quad R(w)$ and $I(w)$ have common real roots, i.e. $\delta(s)$ has roots on the imaginary axis.,

ii) Or $N_{R}^{*}(w)$ and $N_{I}^{*}(w)$ have common real roots, i.e. $N^{*}(s)$ has roots on the imaginary axis.

Since we test the characteristic polynomial $\delta(s)$, the case (i) is only considered.

\section{For a $P$ controller:}

$$
\begin{aligned}
& p(w)=D_{e}\left(-w^{2}\right) N_{e}\left(-w^{2}\right)+w^{2} D_{o}\left(-w^{2}\right) N_{o}\left(-w^{2}\right)+K_{p}\left[N_{e}^{2}\left(-w^{2}\right)+w^{2} N_{o}^{2}\left(-w^{2}\right)\right] \\
& q(w)=w\left[D_{o}\left(-w^{2}\right) N_{e}\left(-w^{2}\right)-D_{e}\left(-w^{2}\right) N_{o}\left(-w^{2}\right)\right]
\end{aligned}
$$

In [2] and [3], the authors gave a computational method for the stabilizing range of feedback gain $K_{p}$. They used a set of strings that are generated to satisfy a certain requirements using the generalized Hermite-Biehler theorem and the generalized Mikhailov criterion respectively. In [1], it was shown that, the number of roots on the imaginary axis of the characteristic polynomial $\delta(s)$ equals the number of the common zeros of the real polynomials $R(w)$ and $I(w)$. The system can be marginally stable or unstable depending on the multiplicity of these imaginary roots taking into consideration that no roots are in the RHP. This last concept will be used in our proposed algorithm, where we write a MATLAB program to run this algorithm.

\section{The Proposed Algorithm:}

1- Obtain the nonnegative real roots of $q(w)$ of multiplicity one.

2- Eliminate the roots of $N^{*}(j w)$ of multiplicity one if any, from the roots obtained in step (1).

3- Equate $p(w)$ to zero using the above obtained nonnegative real roots in step (1) and obtain the unknown parameters $K_{p}$, i.e. $\left[K_{p 0}, K_{p 1}, \quad, K_{p t}\right]$.

4- At these values of $K_{p}$, the system is marginally stable or unstable depending on the presence of the roots of $\delta(s)$ in the right half plane (RHP). This can be checked by substituting these values of $K_{p}$ in $\delta(s)$ and obtain the roots or 
obtaining the argument of $\delta(s)$ i.e. $\theta(w)$ as $w$ approaches infinity. If $\delta(s)$ has no roots in the RHP, then $\theta(w \rightarrow \infty)=n \pi / 2$.

5- Sort these values of $K_{p}$ that make the system on the margin of stability in ascending order $\left(\left[K_{p 0}, K_{p 1}, \quad, K_{p t}\right]\right.$, where $\left.K_{p 0}<K_{p 1}<<K_{p t}\right)$ and check the stability of $\delta(s)$ at $\left(K_{p 0}-\varepsilon\right)$ and $\left(K_{p t}+\varepsilon\right)$ (where $\varepsilon$ is a small positive number) to determine if $\left(-\infty, K_{p 0}\right)$ and $\left(K_{p t}, \infty\right)$ are in the stability ranges or not.

\section{- For a stabilizing PI controller}

$$
G_{c}(s)=\frac{K_{i}+K_{p} s}{s}
$$

then

$$
\delta(s)=s D(s)+\left(K_{i}+K_{p} s\right) N(s)
$$

As in the case of $\mathrm{P}$ controller,

where

$$
\delta(j w) N^{*}(j w)=p(w)+j q(w)
$$

$$
\begin{aligned}
& p(w)=p_{1}(w)+K_{i} p_{2}(w), \\
& q(w)=q_{1}(w)+K_{p} q_{2}(w),
\end{aligned}
$$

and

$$
\begin{aligned}
& p_{1}(w)=-w^{2}\left(D_{o}\left(-w^{2}\right) N_{e}\left(-w^{2}\right)-D_{e}\left(-w^{2}\right) N_{o}\left(-w^{2}\right)\right) \\
& p_{2}(w)=N_{e}\left(-w^{2}\right) N_{e}\left(-w^{2}\right)+w^{2} N_{o}\left(-w^{2}\right) N_{o}\left(-w^{2}\right) \\
& q_{1}(w)=w\left(D_{e}\left(-w^{2}\right) N_{e}\left(-w^{2}\right)+w^{2} D_{o}\left(-w^{2}\right) N_{o}\left(-w^{2}\right)\right) \\
& q_{2}(w)=w\left(N_{e}\left(-w^{2}\right) N_{e}\left(-w^{2}\right)+w^{2} N_{o}\left(-w^{2}\right) N_{o}\left(-w^{2}\right)\right)
\end{aligned}
$$

From these expressions [3], we note that $K_{i}, K_{p}$ appear in $p(w)$ and $q(w)$ respectively. Moreover, for every fixed $K_{p}$, the roots of $q(w)$ do not depend on $K_{i}$, and we use the same algorithm presented in the case of P controller (using $K_{i}$ instead of $K_{p}$ ). Thus, by sweeping over all real $K_{p}$ and solving a constant gain stabilization problem (stabilizing $\mathrm{P}$ controller problem) for each value, we can determine the set of all stabilizing values $\left(K_{i}, K_{p}\right)$ for a given plant.

However, there is no need to sweep over all real values of $K_{p}$. From [3] a necessary condition for the existence of a stabilizing $K_{i}$ value at a fixed value of $K_{p}$ is that the number of real, non-negative, distinct finite roots of odd multiplicities of $q(w)$ be at least

$$
\frac{|n-(l(N(s))-r(N(s)))|}{2} \quad \text { if } m+n \text { is even }
$$

or 


$$
\frac{|n-(l(N(s))-r(N(s)))|+1}{2} \quad \text { if } m+n \text { is odd }
$$

where

$n=$ degree of $\delta(s)$

$m=$ degree of $N(s)$

$l(N(s))=$ number of the roots of $N(s)$ in the LHP.

$r(N(s))=$ number of the roots of $N(s)$ in the RHP.

These ranges of $K_{p}$ that satisfy the condition can be determined by applying the root locus ideas which is presented in [3].

\section{- For a stabilizing PID controller}

$$
\begin{aligned}
G_{c}(s) & =\frac{K_{i}+K_{p} s+K_{d} s^{2}}{s}, \\
\delta(s) & =s D(s)+\left(K_{i}+K_{p} s+K_{d} s^{2}\right) N(s)
\end{aligned}
$$

then,

where

$$
\delta(j w) N^{*}(j w)=p(w)+j q(w)
$$

$$
\begin{aligned}
& p(w)=p_{1}(w)+\left(K_{i}-K_{d} w^{2}\right) p_{2}(w) \\
& q(w)=q_{1}(w)+K_{p} q_{2}(w)
\end{aligned}
$$

and

$$
\begin{aligned}
& p_{1}(w)=-w^{2}\left(D_{o}\left(-w^{2}\right) N_{e}\left(-w^{2}\right)-D_{e}\left(-w^{2}\right) N_{o}\left(-w^{2}\right)\right) \\
& p_{2}(w)=N_{e}\left(-w^{2}\right) N_{e}\left(-w^{2}\right)+w^{2} N_{o}\left(-w^{2}\right) N_{o}\left(-w^{2}\right) \\
& q_{1}(w)=w\left(D_{e}\left(-w^{2}\right) N_{e}\left(-w^{2}\right)+w^{2} D_{o}\left(-w^{2}\right) N_{o}\left(-w^{2}\right)\right) \\
& q_{2}(w)=w\left(N_{e}\left(-w^{2}\right) N_{e}\left(-w^{2}\right)+w^{2} N_{o}\left(-w^{2}\right) N_{o}\left(-w^{2}\right)\right)
\end{aligned}
$$

From these expressions [3], we note that $K_{i}, K_{d}$ appear in $p(w)$ while $K_{p}$ appears in $q(w)$. Moreover, for every fixed $K_{p}$, the roots of $p(w)$ will depend on both $K_{i}$ and $K_{d}$. Since there are two unknown variables in $p(w)$, a linear programming problem has to be solved for each fixed gain $K_{p}$, where every two equations are solved and the stability of the solution points of all two equations are checked via the roots types of $\delta(s)$ or the argument $\theta(w)$ as $w$ approaches infinity, so a modification is made on our proposed algorithm. The ranges of $K_{p}$ can be obtained as discussed in the PI controller.

\section{Modified Proposed Algorithm (at every fixed $K_{p}$ ):}

1- Obtain the nonnegative real roots of $q(w)$ of multiplicity one.

2- Eliminate the roots of $N^{*}(j w)$ of multiplicity one if any from the roots obtained in step (1). 
3- Equate $p(w)$ to zero using the above obtained nonnegative real roots in step (1). Since there are two unknown variables $\left(\left(K_{i}, K_{d}\right)\right)$ in $p(w)$, a linear programming problem has to be solved for each fixed gain $K_{p}$.

4- At these set of $\left(K_{i}, K_{d}\right)$ values, the system is marginally stable or unstable depending on the presence of the roots of $\delta(s)$ in the RHP. This can be checked by substituting these values in $\delta(s)$ and obtain the roots or obtaining the argument of $\delta(s)$ i.e. $\theta(w)$ as $w$ approaches infinity. If $\delta(s)$ has no roots in the RHP, then $\theta(w \rightarrow \infty)=n \pi / 2$.

5- Since the equations is linear, so the set of $\left(K_{i}, K_{d}\right)$ values that make the system on the margin of stability will form boundaries of a convex polygon or an intersection of half planes.

In the following section, we will illustrate the suggested method by an example presented in [3].

\section{EXAMPLE}

Given the plant described by the rational transfer function $G(s)=\frac{N(s)}{D(s)}$, where

$$
\begin{aligned}
& N(s)=s^{3}-4 s^{2}+s+2 \\
& D(s)=s^{5}+8 s^{4}+32 s^{3}+46 s^{2}+46 s+17
\end{aligned}
$$

We will consider the three types of controllers (P, PI and PID controller).

\section{1- For a P controller}

$$
G_{c}(s)=K_{p}
$$

The closed-loop characteristic polynomial as in Eq.(1) is

$$
\delta(s)=D(s)+K_{p} N(s)
$$

Write

where

$$
\begin{aligned}
& N(s)=N_{e}\left(s^{2}\right)+s N_{o}\left(s^{2}\right) \\
& D(s)=D_{e}\left(s^{2}\right)+s D_{o}\left(s^{2}\right)
\end{aligned}
$$

$$
\begin{gathered}
N_{e}\left(s^{2}\right)=-4 s^{2}+2 \\
N_{o}\left(s^{2}\right)=s^{2}+1 \\
D_{e}\left(s^{2}\right)=8 s^{4}+46 s^{2}+17 \\
D_{o}\left(s^{2}\right)=s^{4}+32 s^{2}+46
\end{gathered}
$$

and, $N^{*}(s)=N(-s)=N_{e}\left(s^{2}\right)-s N_{o}\left(s^{2}\right)=\left(-4 s^{2}+2\right)-s\left(s^{2}+1\right)$

Then we have

$$
\begin{gathered}
N_{e}\left(-w^{2}\right)=4 w^{2}+2 \\
N_{o}\left(-w^{2}\right)=-w^{2}+1
\end{gathered}
$$




$$
\begin{aligned}
& D_{e}\left(-w^{2}\right)=8 w^{4}-46 w^{2}+17 \\
& D_{o}\left(-w^{2}\right)=w^{4}-32 w^{2}+46
\end{aligned}
$$

Now we construct the polynomial,

$$
\delta(j w) N^{*}(j w)=p(w)+j q(w)
$$

where $p(w), q(w)$ are obtained from Eq.(5) and Eq.(6)

$$
\begin{aligned}
& p(w)=-w^{8}+\left(65+K_{p}\right) w^{6}+\left(14 K_{p}-246\right) w^{4}+\left(22+17 K_{p}\right) w^{2}+\left(34+4 K_{p}\right) \\
& q(w)=12 w^{7}-180 w^{5}+183 w^{3}+75 w
\end{aligned}
$$

\section{We apply the proposed algorithm}

1- The nonnegative real roots of $q(w)=0$ of multiplicity one are :

$$
w_{0}=0, w_{1}=1.2018, w_{2}=3.7240 \text {. }
$$

2- There are no roots of $N^{*}(s)$ on the $j w$-axis.

3- For the above obtained roots in step (1), equate $p(w)$ to zero and obtain the unknown parameters $K_{p}$. The calculated gain constants $K_{p}$ are: $\left[K_{p 0}=-8.5, K_{p 1}=4.2109, K_{p 2}=-15.9665\right]$.

4- At these values of $K_{p}$, we check the stability of the characteristic polynomial $\delta(s)$ of Eq.(1).

a. For $K_{p 0}=-8.5, p(w)=q(w)=0$ and $\delta(s)$ has a root at the origin and the system is unstable and $\theta(w \rightarrow \infty)=5 \pi / 2$.

b. For $K_{p 1}=4.2109, p(w)=q(w)=0$ and $\delta(s)$ has two pure imaginary roots $\pm j 1.2018$ and the system is marginally stable as $\delta(s)$ has no roots in the RHP and $\theta(w \rightarrow \infty)=5 \pi / 2$.

c. For $K_{p 2}=-15.9665, p(w)=q(w)=0$ and $\delta(s)$ has two pure imaginary roots $\pm j 3.724$ and the system is unstable as $\delta(s)$ has a positive real root. It is found that, $\theta(w \rightarrow \infty)=4 \pi / 2 \neq 5 \pi / 2$.

5- To find the range of $K_{p}$ for a stable system, the system stability is checked at $K_{p}=-8.5+\varepsilon$ where $\varepsilon$ is a small positive number and it was stable.

Thus the range of $K_{p}$ is $-8.5<K_{p}<4.2109$.

\section{2- For a PI controller}

$$
G_{c}(s)=\frac{K_{i}+K_{p} s}{s}
$$

The closed-loop characteristic polynomial as in Eq.(7) is

$$
\delta(s)=s D(s)+\left(K_{i}+K_{p} s\right) N(s)
$$

Now we construct the polynomial,

$$
\delta(j w) N^{*}(j w)=p(w)+j q(w)
$$


where $p(w), q(w)$ are obtained from Eq.(8) and Eq.(9) by determining the following polynomials as follows:

$$
\begin{aligned}
& p_{1}(w)=-12 w^{8}+180 w^{6}-183 w^{4}-75 w^{2} \\
& p_{2}(w)=w^{6}+14 w^{4}+17 w^{2}+4 \\
& q_{1}(w)=-w^{9}+65 w^{7}-246 w^{5}+22 w^{3}+34 w \\
& q_{2}(w)=w^{7}+14 w^{5}+17 w^{3}+4 w
\end{aligned}
$$

then

$$
\begin{aligned}
& p(w)=p_{1}(w)+K_{i} p_{2}(w) \\
& q(w)=q_{1}(w)+K_{p} q_{2}(w)
\end{aligned}
$$

Using the root locus ideas [3] to satisfy the condition of Eq.(11), the range of $K_{p}$ values can be narrowed down to $K_{p} \in(-8.5,4.2333)$.

By sweeping over different $K_{p}$ values in this interval and following the algorithm illustrated above as in P controller (but for $K_{i}$ instead of $K_{p}$ ), we can generate the set of $\left(K_{p}, K_{i}\right)$ values for which $\delta(s)$ is stable. This set is sketched in Fig.(2).

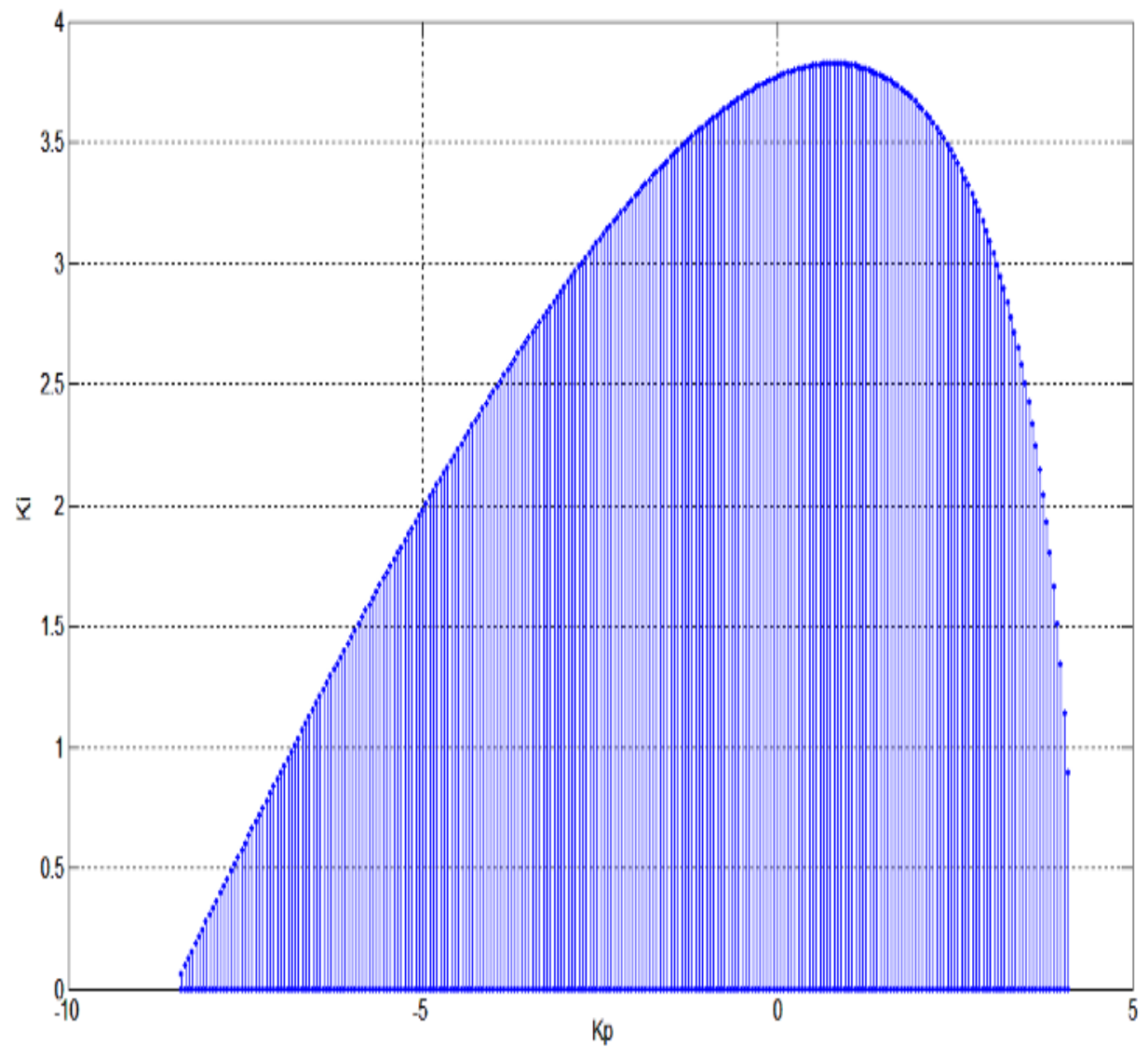

Fig. (2) The stabilizing set of $\left(\mathrm{K}_{\mathrm{p}}, \mathrm{K}_{\mathrm{i}}\right)$ values 


\section{For a PID controller}

$$
G_{c}(s)=\frac{K_{i}+K_{p} s+K_{d} s^{2}}{s}
$$

The closed-loop characteristic polynomial as in Eq.(12) is

$$
\delta(s)=s D(s)+\left(K_{i}+K_{p} s+K_{d} s^{2}\right) N(s)
$$

Now we construct the polynomial,

$$
\delta(j w) N^{*}(j w)=p(w)+j q(w)
$$

where $p(w), q(w)$ are obtained from Eq.(13) and Eq.(14) by using the same polynomials that was determined in the PI controller, then

$$
\begin{aligned}
& p(w)=p_{1}(w)+\left(K_{i}-K_{d} w^{2}\right) p_{2}(w) \\
& q(w)=q_{1}(w)+K_{p} q_{2}(w)
\end{aligned}
$$

Using the root locus ideas [3] to satisfy the condition of Eq.(8) as in the PI controller, the range of $K_{p}$ values can be narrowed down to $K_{p} \in(-8.5,4.2333)$.

By sweeping over different $K_{p}$ values in this interval and following the modified algorithm illustrated before, we can generate the set of $\left(K_{p}, K_{i}, K_{d}\right)$ values for which $\delta(s)$ is stable.

\section{We apply the modified algorithm (for every fixed $K_{p}$ ):}

1- Obtain the nonnegative real roots of $q(w)$ of multiplicity one.

2- There is no roots of $N^{*}(s)$ on the $j w$-axis.

3- Equate $p(w)$ to zero using the above obtained nonnegative real roots in step (1). Since there are two unknown variables $\left(\left(K_{i}, K_{d}\right)\right)$ in $p(w)$, a linear programming problem has to be solved for each fixed gain $K_{p}$. These equations are:

$p_{1}\left(w_{0}\right)+\left(K_{i}-K_{d} w_{0}^{2}\right) p_{2}\left(w_{0}\right)=0$

$p_{1}\left(w_{1}\right)+\left(K_{i}-K_{d} w_{1}^{2}\right) p_{2}\left(w_{1}\right)=0$

$p_{1}\left(w_{2}\right)+\left(K_{i}-K_{d} w_{2}^{2}\right) p_{2}\left(w_{2}\right)=0$

$p_{1}\left(w_{t}\right)+\left(K_{i}-K_{d} w_{t}^{2}\right) p_{2}\left(w_{t}\right)=0$

4- At these set of $\left(K_{i}, K_{d}\right)$ values, the system is marginally stable or unstable depending on the presence of the roots of $\delta(s)$ in RHP. This can be checked by substituting these values in $\delta(s)$ and obtain the roots or obtaining the argument 
of $\delta(s)$ i.e. $\theta(w)$ as $w$ approaches infinity. If $\delta(s)$ has no roots in the RHP, then $\theta(w \rightarrow \infty)=n \pi / 2$.

5- $\quad$ Since the equations are linear, so the set of $\left(K_{i}, K_{d}\right)$ values that make the system on the margin of stability will form boundaries of a convex polygon. The set is shown in Fig.(3).

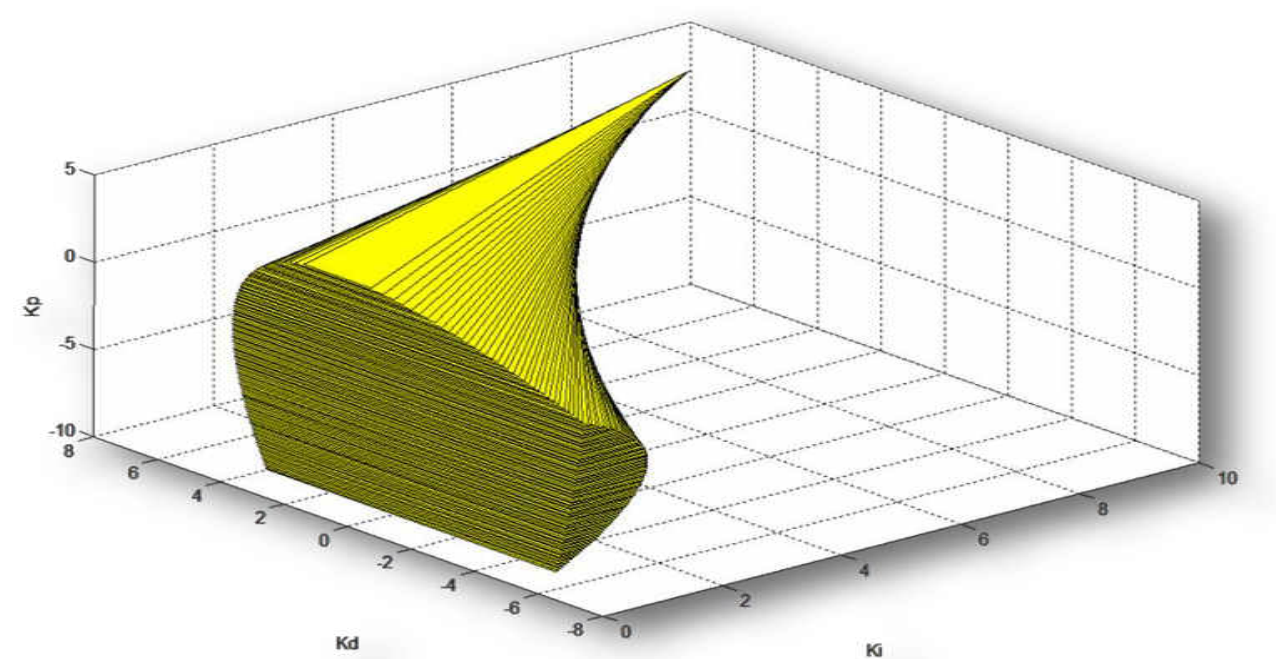

Fig. (3) The stabilizing set of $\left(\mathrm{K}_{\mathrm{p}}, \mathrm{K}_{\mathrm{i}}, \mathrm{K}_{\mathrm{d}}\right)$ values

In this paper we use the generalized Mikhailov criterion to find a solution to the problem of feedback stabilization of a given linear-time invariant plant using a $\mathrm{P}$ controller, a PI controller and a PID controller. In [1], it was shown that, the number of roots on the imaginary axis of the characteristic polynomial $\delta(s)$ equals the number of the common zeros of the real polynomials $R(w)$ and $I(w)$. The system can be marginally stable or unstable depending on the multiplicity of these imaginary roots. We proposed an algorithm using MATLAB software using the last concept. An example with three types of controllers is given to illustrate the proposed algorithm to define the controllers gains limit.

\section{REFERENCES}

[1] A. I. Saleh, M. M. Hasan and N. M. M. Darwish, "The Mikhailov Stability Criterion Revisited" Submitted for publication.

[2] L. H. Keel and S. P. Battacharyya, "A Generalization of Mikhailov's Criterion with Applications" Proceedings of the American Control Conference, pp. 4311-4315, Chicago, June 2000.

[3] G. J. Silva, A. Datta and S. P. Bhattacharyya, "PID Controllers for Time-Delay Systems", Birkhauser, Boston, 2005 


\section{تحديا مدى معاملات المحكم باستخدام نظرية ميخايلوف للاتزلن}

تقيد نظرية ميخايلوف أن المعادلة الواصفة للنظام من الدرجة ن لكى تكون "هوريفتز" يجب أن تلتخير

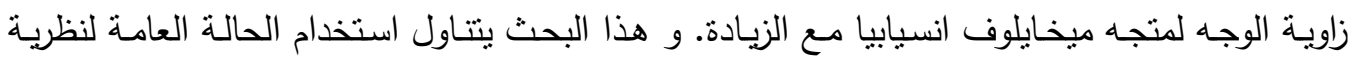

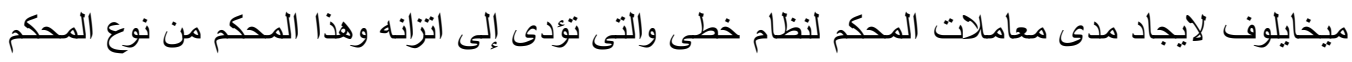

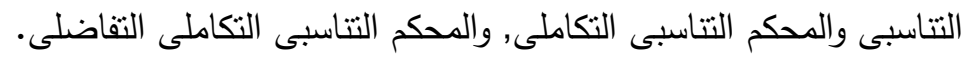

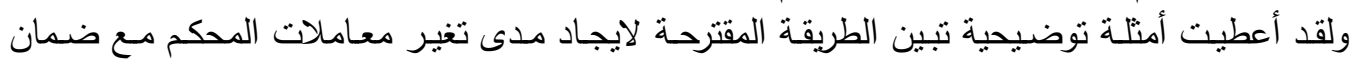
الاتزان. 